\title{
Lessons Learnt From Operating ESEO Educational Spacecraft
}

\author{
G.Curzi*, A. Lucci, A. Locarini, D. Modenini, P. Tortora \\ Department of Industrial Engineering \\ University of Bologna \\ Via Fontanelle 40, I-47121, Forlì (FC) - Italy \\ *giacomo.curzi2@unibo.it \\ G. Mariotti, D. Cinarelli, V. Fabbri, N. Melega \\ SITAEL S.p.A. \\ Via Guarini 13, I-47121, Forlì (FC) - Italy
}

\begin{abstract}
Since 2003, the Microsatellite and Space Microsystems Lab at the University of Bologna (UniBo) has extended his research activities to the design of a ground segment for small satellites missions. In the framework of the European Student Earth Orbiter (ESEO), an ESA Education Office project for the development of a microsatellite mission, with SITAEL S.p.A. as the Industrial System Prime Contractor, the firstgeneration GS has been upgraded to support ESEO operations. UniBo was in charge for the design and development of the Mission Control Centre (MCC), the implementation of the primary ground station for telemetry and telecommand operations, and of the secondary one for the downlink of payloads data. ESEO was launched on December $3^{\text {rd }}$, 2018. Soon after launch, the activities planned for the Launch and Early Orbit Phase (LEOP) were forced to be held back due to the lack of success in commanding the spacecraft. After weeks of coordinated efforts among spacecraft operators, spacecraft engineers and ESA technical staff, LEOP activities could be resumed, thanks to the support of the radio amateur community and of ES5PC ground station in Tartu. Since then, new challenges are coming, which need to be faced for ESEO mission to advance further: this paper provides a thorough perspective of the achievements and lessons learnt during these months of operations.
\end{abstract}

\section{Keywords: Ground segment, Flight Operations, ESEO}

\section{INTRODUCTION}

The European Student Earth Orbiter (ESEO) is a project of the Education Office of the European Space Agency (ESA) aimed at providing students with unparalleled hands-on experience to help preparing qualified space engineers for the Europe's future [1]. This is achieved through the design, development, integration and testing of a micro-satellite to be launched in a sun-synchronous low Earth orbit. The objectives of the mission are: taking pictures of the Earth for educational outreach purposes, providing dosimetry and space plasma measurement, and testing technologies for future education satellite missions. SITAEL is the prime contractor and responsible for the ESEO platform and mission implementation whilst the university network provides the scientific and technological payloads and develops the ground segment.
UniBo team is in charge of the design and development of the mission control center as well as the implementation of the primary ground station (for TeleMetry and TeleCommand TMTC - operations), and of the secondary one (for the downlink of payloads data).

ESEO was launched on December $3^{\text {rd }}, 2018$ : soon after launch, the roadmap originally conceived for the operating the spacecraft had to be re-planned to overcome some unexpected issues.

This paper offers an overview of the efforts towards the operational phase of ESEO mission, describing the issues encountered, investigations performed, solutions and/or workaround identified. The manuscript is organized as follows: the next section includes details about the ground segment implemented for ESEO operations. Afterwards, the activities performed in order to get ready for launch are presented focusing on the preparation and testing of the flight procedures. The following two sections cover LEOP and commissioning, with emphasis on the tools which revealed useful during critical phases, such as distributing the ground segment and setting up a tool for telemetry visualization/collection from multiple sources. Finally, conclusions are drawn, together with the lessons learnt so far.

\section{ESEO GROUND SEGMENT}

The primary TMTC ground station operates in the UltraHigh Frequency (UHF) band for uplink and downlink. It consists of two Yagi antennas: one, in the amateur band (430$440 \mathrm{MHz}$ ), dedicated to the ESEO satellite and another, operating in the commercial UHF band (400 MHz), for future applications. With respect to the first ground station implementation, the current one is designed under the Software-Defined Radio (SDR) paradigm, which allows fast and economical reconfiguration of the ground station since the signal is digitally processed at software level [3].

The Secondary Science Data ground station operates in Sband, and uses a parabolic dish antenna with a septum dish feed. 
A dedicated control room is furnished with technical equipment to support the mission monitoring and control related activities. It hosts three workstations and the Radio Frequency equipment such as switches, High Power Amplifiers and radio interfaces. The first workstation is dedicated to the control of the RF front-end and the Antenna tracking. The second workstation is a spare for future missions and the third workstation is entirely dedicated to the ESEO mission. It hosts the mission control system, which is all the software dedicated to the accomplishment of the ESEO mission, from the database storage to the Spacecraft Monitoring and Control (M\&C) system for commanding the spacecraft and visualizing telemetry.

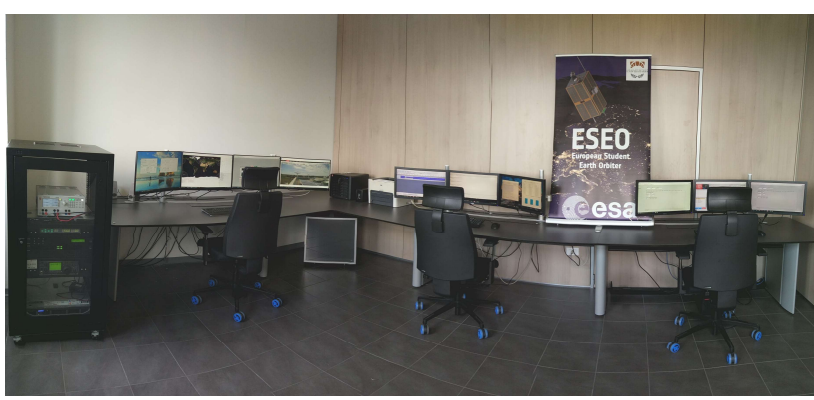

Fig. 1. Mission Control Center at UniBo premises.

The design of the M\&C system allowed UniBo students to develop a key background in the field of ground operations. A great effort has been devoted to design an intuitive and easyto-use graphical user interface, helping the spacecraft operator to handle TMTC data within the short duration of the satellite visibility pass. Specifically, the M\&C system has a threefold purpose: a) the selection of the desired commands to send b) the visualization of the received satellite telemetry data and c) the connection to a mission database for storing the downlinked data and retrieving the various commands and satellite parameters, if necessary.

\section{LAUNCH PREPARATION}

The preparation and test of the flight procedures were the core activity of the University of Bologna during the preparation-to-launch phase. To this end, a list of draft operations in the form of raw commands sequences were provided by SITAEL. These sequences have then been expanded and tailored to the Ground Segment setup, with particular focus on the M\&C Software interface outlining the so-called flight operations procedures (FOP).

A flight procedure is a sequence of operations that the spacecraft operator executes in order to command the spacecraft toward a given state. The desired spacecraft state is thus the objective of the execution the operational procedure. For ESEO, 192 flight procedures were developed to characterize all ESEO's state transitions, both basic and complex, and laying down plans on how to handle anomalies. Each flight procedure is reported on a spreadsheet with the following fields:

- Title;
- Duration: Time needed for execution;

- Criticality: Degree of criticality defines the needed authorization and the personnel to be involved in the planification for the procedure execution;

- Objective: Description of the final state of the spacecraft after the completion of the procedure;

- Introduction: Brief general description and applicability of the procedure;

- Procedure description: step by step operations to be accomplished to achieve the objective (i.e. sequence of set up and commands to be sent from the M\&C System);

- Caution: warnings on the application of the procedure;

- Applicable and reference documents: link to related manuals;

- Related procedures: link to procedures that may be needed during or soon after the current procedures;

- Needed Authorisation (if any).

All flight procedures were tested on the Avionic Test Bench (ATB) to check their effectiveness in leading the spacecraft into the target state. The setup of the test emulates the real spacecraft operational conditions as much as possible. Limitations in matching the real scenario were present though, such as missing attitude sensors and GPS readings, and the absence of doppler shift in the radiofrequency link. A workstation runs the MCS software which interfaces with another workstation operating Software defined radio hardware. All sent commands and received answers were logged into an archiving system as in a real mission scenario.

Along with the flight operational procedures, operational workflows (i.e. lists of actions for the spacecraft operator to perform, arranged in an "event tree" configuration) were agreed with SITAEL on the base of the final ESEO's design. A total of 10 workflows were deemed sufficient for ensuring a safe commissioning and mission operation:

- LEOP START: establish the first contact with the satellite. Three different final states foreseen: No signal, Nominal, Emergency signal;

- LEOP LOST: a set of procedures and crosschecks is suggested before returning to LEOP START;

- LEOP NOMINAL: guides the operator through a series of basic health checks before moving on to the platform commissioning;

- LEOP-S3: provides the operations to bring the spacecraft from an emergency state (in LEOP) to the nominal LEOP state (i.e. LEOP NOMINAL);

- COMMISSIONING START: applied for each platform subsystem, guides the operator to perform specific platform checks and has three possible outcomes: platform Commissioning failed with or without criticality or ok; 
- COMMISSIONING NONCRITICAL: guides the resolution of non-critical failures in platform commissioning toward the payload commissioning;

- COMMISSIONING CRITFAIL: guides the resolution of critical failures in commissioning toward a noncritical condition to continue platform commissioning and then payload commissioning;

- COMMISSIONING PAYLOADS: guides the payloads commissioning. Failure of any payload commissioning is handled internally towards a recovery or final report;

- NOMINAL: guides the nominal operations;

- DISPOSAL: guides the satellite shutdown.

These workflows were then made available to the ground station operators in form of a wall poster. The rationale for employing workflows in addition to the FOPs lies in that the former provide a picture of the mission at higher level, highlighting how each FOP interconnects with the others and possibly having roles in different mission phases. FOPs and workflows together also allow for a comprehensive operations testing and training phase during launch preparation.

\section{LAUCH AND EARLy ORBIT PhaSe}

On December $3^{\text {rd }}, 2018$ at 19:34 CET, the European Earth Student Orbiter was successfully launched into space onboard of a SpaceX Falcon 9 launcher from the Vandenberg Air Force Base in California (USA), as part of the Spaceflight's SSO-A SmallSat Express mission.

The LEOP is the first and the most critical part of any mission. The spacecraft, after a series of vibrations, accelerations and mechanical stresses due to the launch, is exposed to the actual space environment for the first time; the operations team starts interfacing with it in this phase. ESEO mission was no different, it showed several unexpected behaviors which forced the team to readjust the entire operations concept, procedures and processes. Being ready to unpredicted behaviors and reacting fast to them is perhaps the most pivotal lesson learnt from the mission. The following subsections summarize the problems encountered during LEOP and the solutions put in place to overcome them.

\section{A. Decoding ESEO's Telemetry}

The first TM data from ESEO was received on December 3rd 20:25:21 UTC: a type 5 beacon which included the expected values of the Fault Detection Isolation and Recovery (FDIR) parameter and the TMTC telemetries. At the same time the first unexpected event occurred: as the beacons kept arriving to the ground, it become quite clear that primary TMTC ground station was achieving a poor performance in decoding the ESEO packets.

Investigation on the problem revealed the cause of the low downlink capabilities to reside both on a high level of environmental noise at UHF band, and on some improvements needed in the decoding part of the LabView-based SDR SW. After a new SW version developed and released, the downlink performances became more in line with other ground stations.
The new SDR SW was developed using GNU Radio environment, an open-source software development toolkit for signal processing [4], which is widespread among the radio amateur community.

\section{B. ESEO first contact}

Shortly after the launch, ESEO's downlink seemed to work properly but it appeared that the commands coming from the GS were not received by the spacecraft. Several Materials Review Boards (MRB) were held along with ESA's experts and SITAEL's engineers to investigate and solve the issue. Time is a crucial constraint in this phase and actions were taken on different fronts: from the inspection of the RF chain and the M\&C SW, to the check of the antenna pointing. In parallel, other ground stations were contacted, some of them having significant higher EIRP than the primary TMTC one, for establishing a first contact with ESEO. A success in establishing an uplink to ESEO using a higher EIRP could hint toward a desensitization of the onboard receiver. The chronological schedule of the attempts is the following:

- 03/12/2018: Forli’s GS (ESEO Primary TMTC GS);

- 19/12/2018: Vigo's GS (ESEO Secondary TMTC GS);

- 22/01/2019: Munich's GS (Primary Payload data GS);

- 28/01/2019: ES5PC's GS (Amateur station in Estonia).

The first successful contact with ESEO was established during the attempt from ES5PC, an amateur station located in Estonia (Fig. 2). The station, with its $4.5 \mathrm{~m}$ parabolic dish and vacuum tube power amplifier, is capable of delivering $1 \mathrm{~kW} R F$ power, which proved to be enough to uplink TCs to ESEO.

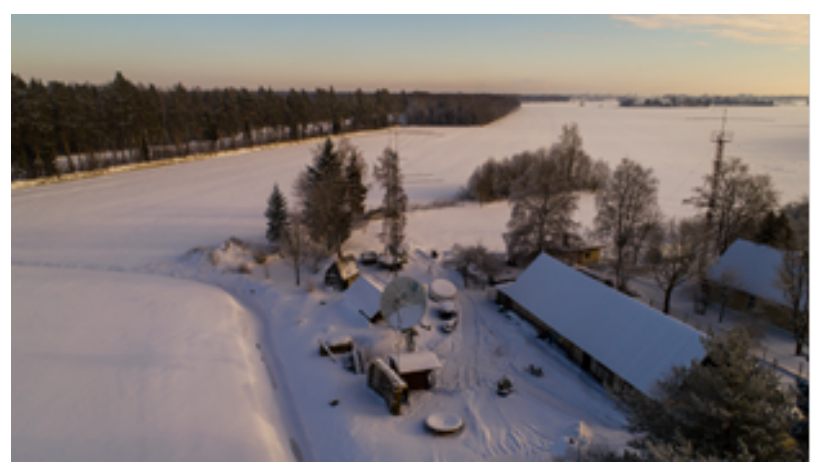

Fig. 2. ES5PC's Amateur Station (ES5PC Photo)

Having a solid network of ground stations is fundamental in a space mission, and coordination among partners is a key factor during LEOP; certainly, this is another lesson learnt from ESEO. The main cause of the desensitization of the ESEO receiver is still unclear.

\section{ESEO Operations}

A reliable link to extend the main TMTC GS has been therefore developed. The extension allowed to replace de facto the antennas and workstation driving the RF front-end at the primary TMTC GS with ES5PC's RF equipment. More 
specifically, a TCP/IP connection was set up for the M\&C workstation being able to send and receive packets to and from Estonia. This was possible thanks to the modular implementation of the Mission Control Center, featuring separated workstations for the M\&C task and for the RF frontend handling task: this resulted to be a convenient layout for setting up a distributed ground segment.

Once the new setup was validated, the LEOP operations could be resumed following the LEOP NOMINAL workflow. During these operations, all the sent commands and any relevant observation during pass were logged on Tracking Pass Reports. Reports were then analyzed with SITAEL in order to agree on the operations for subsequent passages. In parallel, the spacecraft operators worked on the upgrade of Forlì GS HW to raise its transmitted EIRP.

During LEOP, the on-board computer experienced a Single Upset Event which made the temporal separation between beacons change from 60 seconds to 572 making an On-Board Data Handling (OBDH) system reset needed. In addition, the TMTC subsystem experienced an issue with the High-Power Amplifier (HPA): the switching-on of the HPA got slightly delayed with respect to the start of the beacon transmission, resulting into a loss of modulation of the received packets on ground.

Investigations on the anomalous behaviour of the HPA delay were extensively carried out on the ATB searching for the root cause of this behavior. However, there was no success in trying to replicate the same anomaly. Nevertheless, the issue could be overcome by forcing periodically a reset of the TMTC through a dedicated High Priority Command (HPC). The need for periodic TMTC reset slowed down the operations toward towards LEOP completion and led to the addition of a new flight procedure.

The main operations performed from then on were:

- Update Time via Time-Tagged telecommands;

- Update TLE via a set of 10 telecommands;

- OBDH reset via HPC;

- $\quad$ Request of House Keeping (HK) History, HK Pages and TM data;

- Set of several on-board parameters via telecommands (e.g. Magnetorquer Actuator Gains);

- $\quad$ TMTC Main Reset via HPC.

\section{Ground Station Network and Data Visualization}

With ESEO transmitting beacons in the amateur UHF band, the radio amateur community was deeply engaged in collecting as much information as possible on the satellite state. A public database was developed to store telemetry from contributors all around the world. Three alternative ways are provided for contributors to write on this database, namely

- SiDS protocol [6]: collects the packets from the radio amateurs implementing SiDS forwarders;
- SatNOGS: collects the packets from any source which uploads its passes to the website [8][5] (mainly from the radio amateur network);

- Alma Mater Ground Station (AMGS) Forlì: packets decoded from the primary TMTC GS.

Simple and fast data visualization is essential not only given the educational purpose of this project, but also from an operational point of view. Ground segment and operations engineers shall monitor the status of health of the platform continuously and plan actions from the MCC accordingly.

The tool selected to make these data available is GRAFANA, an open source project for visualizing metrics [7]. Supporting integration for several database (MySQL in our case) GRAFANA allows the user to visualize all ESEO telemetries at the following link: http://eseo.ddns.net/. Each parameter (e.g. SOLAR PANEL Temperatures) is represented in a dedicated panel and grouped into several dashboards (e.g. Beacon Power), see Fig. 3.

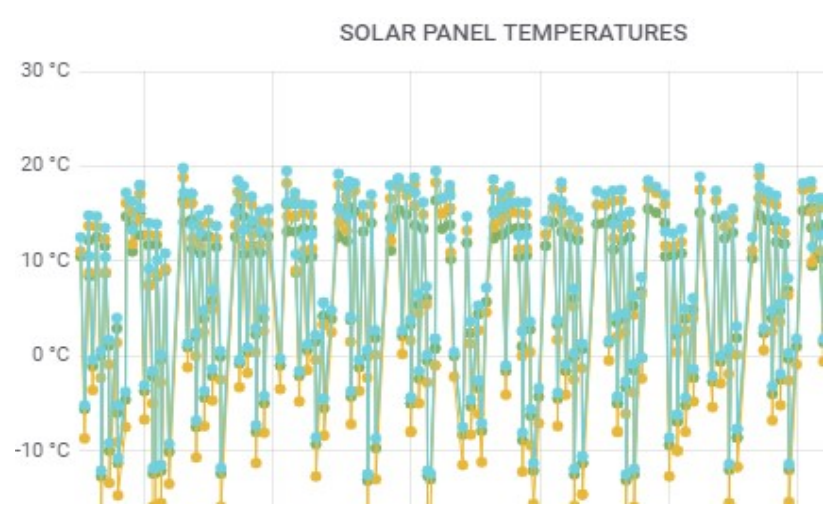

Fig. 3. ESEO Solar Panel Temperatures (source: http://eseo.ddns.net/)

\section{COMMISSIONING}

The Commissioning phase is aimed at running the set of operations and checks needed to declare the satellite ready to begin its nominal mission. For ESEO, one of these operations is the transition from the post-launch spinning attitude to a three-axis stabilized attitude. On 21 March 2019, all the commands needed by the spacecraft to determine its orbital position and attitude were uploaded on-board.

During this phase, an off-nominal attitude of ESEO was detected from its telemetry. Following the same approach as for other non-conformity, an investigation started in coordination with MRB meetings. This gave rise to a list of actions to be performed in the following passes, namely: i) the nominal beacon rotation was forced to AOCS beacon only, to collect all possible information on attitude; ii) magnetometers and magnetorquers data were analysed in detail through dedicated telecommands and iii) to check the output of the onboard International Geomagnetic Reference Field (IGRF) model, which was as expected. Despite the nominal attitude was not achieved, other actions to advance ESEO mission educational outreach were undertaken, which are described in the following. 


\section{A. AMSAT UK Activation}

In this non-nominal yet safe condition, the activation of the AMSAT-UK Communication System was greenlighted, to mark the start of the educational payloads commissioning. Developed by University of Surrey (UK), this payload provides a continuous stream of satellite and science data for educational purposes and it is the first amateur payload to use L-band communication. Therefore, switching on this payload had also the benefit of providing independent source of telemetry data.

On April 12 $2^{\text {th }}, 2019$, AMSAT-UK started operating nominally. From 10:43 UTC to 14:18 UTC, 270 valid packets were received from the U.K., Japan, Australia and Brazil. All payload telemetry channels were functional and operative within valid ranges; all telemetry generated from the payload itself was consistent with pre-delivery testing [9].

AMSAT-UK was unexpectedly shut down by an OBDH reset occurred during an unknown ESEO state of continuous transmission (see sub-section B).

\section{B. Latest developments: rejection train}

Roughly one orbit later, at about 12:17 UTC, ESEO started transmitting rejection (REJ) messages continuously, one packet per second. Since no telecommands were transmitted to ESEO during this orbit, due to a lack of visibility from the uplink ground station (ES5PC's station), the possibility that the behavior was triggered from ground was soon ruled out. Investigations on the TMTC SW and tests on the ATB performed by SITAEL led to the hypothesis of self-oscillation at TMTC level: $\mathrm{S} / \mathrm{C}$ is transmitting to itself REJ responses triggered by its own transmitted packets. It is unclear however what may have triggered this auto-excitation, and whether this may be linked to the interaction between AMSAT and the platform RF system (although pre-launch EMC tests showed no signs of such a risk).

During this period, ESEO experienced an automatic transition from mode 3 (Damping and Safe) to mode 4 (AOCS Normal Sun/Eclipse-Nominal) and from mode 4 to safe mode 2 (S2) due to a malfunction of the MWM (set "not reliable" and switched off).

Several unsuccessful attempts were performed to unlock ESEO from this uncontrollable state, both from the ES5PC ground station and from another amateur station, I1NDP, in Italy. None of them was successful: at the moment of writing, ESEO is still transmitting one REJ every one second, which prevents any further advancement towards mission completion since the RF system is not full duplex and cannot be commanded while transmitting. Nevertheless, ESEO keeps transmitting also beacons, which allows for continuous monitoring of its status of health. The data in these beacons shows that nine months after launch the power system (solar arrays, batteries, power management and power delivery boards), the OBDH and the RF transmit chain are operating nominally showing no signs of degradation.

\section{CONCLUSIONS}

After almost 9 months in orbit, the in-flight experience gathered with ESEO spacecraft unveiled many challenges.

A first lesson learnt from operating the spacecraft is the strong need for flexibility and responsiveness to unexpected problems. Nevertheless, each unpredicted issue shall be considered as a chance of professional growth.

Working closely with ESA's experts and SITAEL's engineers has been an invaluable source of technical knowledge for the university team. Planning ESEO passes through daily updates with system engineers and reviewing the state of the spacecraft through weekly meetings with ESA were essential in order to understand how to recognize and prevent anomalies, how to identify a problem and how to deal with it.

Management of time and resources proved to be pivotal for the success of ground operations, since many tasks must be performed in the very short time frame set by the satellite visibility over the ground station, allowing also for contingencies. A solid ground station network supporting the mission is mandatory. Coordination among partners is fundamental in a critical phase as the LEOP is; this is a lesson certainly learnt from operating ESEO.

\section{ACKNOWLEDGMENT}

The authors wish to acknowledge the support from ESA Educational Office. UniBo team is especially grateful to the personnel of the entire network of ground stations involved during ESEO operations, in particular, University of Vigo team, TU Munich team, ES5PC and Tartu Observatory team, and I1NDP. The contribution of the radio-amateur community in general, through the SATGNOS platform, is also acknowledged.

\section{REFERENCES}

[1] Bruzzi D., et al. The ESEO development: merging technical with educational challenges, Proceedings of the Small Satellites Systems and Services - The 4S Symposium, European Space Agency, Majorca, Spain, 26-30 May 2014

[2] P. Tortora e E. Troiani, "The microsatellite program at Università di Bologna" Acta Astronautica, vol. 56, pp. 696-704, 2005.

[3] D. Bruzzi, P. Tortora, F. Giulietti, P. Galeone e A. De Luca, "The ESEO development: merging technical with educational challenges" in The $4 S$ Symposium, Spain: Majorca, 2014.

[4] GNU Radio Online Documentation: https://wiki.gnuradio.org.

[5] M. Bosco, P. Tortora e D. Cinarelli, "Alma Mater Ground Station transceiver: A software defined radio for satellite communications", 2014 IEEE Metrology for Aerospace, pp. 549-554, 2014.

[6] S. Dombrovski, "Simple Downlink Share Convention v0.9 (SiDS)", 15 January 2015. [Online]. https://picsat.obspm.fr/sids/doc?locale=en. [Accessed: 04 August 2019].

[7] GRAFANA Online Documentation: https://grafana.com/docs/.

[8] "SatNOGS", Libre Space Foundation, [Online]. Available: https://satnogs.org/. [Accessed: 04 August 2019].

[9] ESEO, AMSAT Payload Preliminary Internal Report, 15.04.2019. 